\title{
La formación dual: un nuevo enfoque de la formación profesional
}

\author{
Iris Molina \\ Departamento de Conocimiento y FP, de Foment del Treball Nacional
}

Resumen: A pesar de los continuos esfuerzos en nuestro país por concebir el sistema de formación profesional con un enfoque integrador, tal como aconseja la Unión Europea, se está lejos de conseguirlo. Por ello, las tasas de desempleo juvenil se mantienen altas y el desempleo de larga duración se perpetúa. El problema de fondo de concebir el sistema de formación profesional de forma fragmentada al atender a la edad de los beneficiarios, a la relación de estos con el mercado laboral o a la Administración, a quien le corresponde la competencia en cada caso, es la ineficacia del propio sistema. El sistema de formación profesional se organiza como un paso previo al trabajo. El requisito sine qua non para conseguir su eficacia es que emane de las empresas, del propio mercado laboral. Son las empresas las que pueden determinar cuáles son sus necesidades reales de formación y qué competencias deben adquirir los futuros profesionales.

La formación profesional dual es una modalidad de oferta diferente que cubre defectos del sistema y consigue, por su propia naturaleza, el acercamiento entre el sistema de formación profesional y las empresas. Resuelve temporalmente problemas del sistema de formación profesional que tendrian que abordarse de forma más decidida. Los esfuerzos que se lleven a cabo en la formación profesional dual deberían ser para que su implantación fuera de calidad, atendiendo sobre todo a sus resultados cualitativos, sin desmerecer los cuantitativos.

Palabras clave: Europa 2020; formación profesional; formación dual; acreditación; competencias. 
The dual training, a new approach to vocational education and training

Abstract: Despite the continuing efforts in our country to conceive the Vocational Education and Training System with an integrative approach, as recommended by European Union, we are a long way from achieving it. For that reason, youth unemployment rates remain high and long term unemployment is perpetuated. The basic problem of conceiving the Vocational Education and Training System in a fragmented way, according to age of the beneficiaries, to the relationship of them with the labor market or to the corresponding Administration in each case, is the inefficiency of the System itself. The Vocational Education and Training System is organized as a first step towards work. The essential prerequisite for its effectiveness is that it should flow from companies, from the labor market itself. The companies can determine which their real training needs are and which competences future professionals need to acquire.

Dual Vocational Education and Training is a different modality of offer that is covering the failings of the system, achieving, by its nature, a closer relationship between the Vocational Education and Training System and the companies. Temporarily it is resolving problems of the Vocational Education and Training System that should be addressed directly. And the efforts made in Dual Vocational Education and Training should be done in order to achieve a high-quality implementation, mainly based on their qualitative results.

Keywords: Europe 2020; Vocational Education and Training; Dual Training; accreditation; skills 


\section{Un nuevo enfoque de la formación profesional}

\section{$1.1 \mathrm{El}$ punto de partida}

La comunicación de la Comisión Europa 2020 (2010:22) Una estrategia para un crecimiento inteligente, sostenible e integrador, aparte de fijar unos objetivos medibles que los estados deberían alcanzar en un tiempo determinado, también establece una serie de directrices para encaminarse hacia ellos, analizando el contexto actual y las previsiones de necesidades perentorias de la sociedad.

En cuanto al tema de la educación predice lo siguiente (Comisión Europea, 2010: 22):

- «En 2020, un total de 16 millones de puestos de trabajo suplementarios requerirán cualificaciones altas, mientras que la demanda de cualificaciones bajas caerá en 12 millones».

- «Prolongar la vida laboral también conllevará la posibilidad de adquirir y desarrollar permanentemente nuevas cualificaciones».

- «Existe un gran riesgo de que personas alejadas del mercado laboral o con débiles vínculos con el mismo los pierdan definitivamente».

En estos momentos la sociedad no está preparada para dar respuesta a estas predicciones y así lo sugiere este documento cuando define el contexto actual (Comisión Europea, 2010: 22):

- «Alrededor de 80 millones de personas solo tienen unas cualificaciones bajas o básicas, pero el aprendizaje a lo largo de la vida beneficia sobre todo a los más formados».

- «Los jóvenes se han visto especialmente afectados por la crisis, con una tasa de desempleo superior al $21 \% »$.

Otros documentos, como El sistema educatiu a Catalunya. Recuperar el prestigi social (2014), en su presentación, refuerzan la idea de la desigualdad formativa existente en la actualidad en nuestro país al poner de manifiesto la estructura inversa, en cuanto a cualificaciones de la población activa, con respecto a la media europea. En Europa, la mayoría de la población tiene unas cualificaciones intermedias, lo que se traduce en que la población activa en España está poco formada y la tasa de abandono escolar es muy alta. No obstante, en comparación con la media europea, la tasa de población con una formación superior es elevada, lo que provoca que haya una población ocupada subempleada, es decir, que ocupa puestos de trabajo para los que no es necesaria la formación adquirida. 


\subsection{La solución}

El sistema de formación profesional debe ser lo suficientemente eficaz para resolver estos problemas y encaminarnos hacia las soluciones que la comparación entre la situación previsible y la actual deja entrever.

En este contexto, la formación se convierte en una necesidad urgente para las personas, las empresas y la sociedad en general.

No obstante, se considera que el sistema de formación profesional español adolece de un problema grave y que de él se derivan una serie de defectos que imposibilitan que esta situación cambie.

Este grave problema es que, a pesar de las repetidas recomendaciones de la Unión Europea para abordar la formación de las personas a lo largo de la vida, el cambio no se ha hecho efectivo en el sistema de formación profesional español: sigue concibiéndose el sistema de forma fragmentada. El sistema de formación profesional está segmentado y se basa en el momento vital que atraviesan las personas en relación con su edad o con el mercado de trabajo en cada momento. Así, se habla de formación reglada u obligatoria para referirse a la FP inicial, que se interpreta que está dirigida a jóvenes de entre 16 y 20 años, aunque cada vez más acceden a ella personas de rangos de edad superiores; a la formación continua, en el caso de que la persona esté trabajando; y a la formación ocupacional, en el caso de que esté desempleada. Incluso, hay varias nomenclaturas que tienen presente la forma de financiación: por ejemplo, la formación continua de oferta o de demanda.

De esta concepción del sistema de formación profesional se derivan unos defectos que lastran el avance y los buenos resultados de la FP:

- La diferenciación entre si la persona trabaja o no tiene como consecuencia sacar a las empresas de la parte de la formación llamada inicial o reglada, lo que hace que se olvide que las personas que están recibiendo esa formación acabarán trabajando en empresas o montando su propia empresa, y que las competencias que están recibiendo son profesionales. Así, la consecuencia inmediata es obviar cualquier asociación entre los centros educativos y las empresas, lo que desvincula la formación profesional del lugar en el que tendría que estar: en el trabajo. Si no se forma para cubrir las necesidades reales de las empresas, el sistema no tiene sentido.

- No hay objetivos estratégicos. Si la finalidad última del sistema de formación profesional es formar, tendría que haber unos objetivos primarios que inspirasen todo el sistema. Si cada subsistema de formación tiene unos objetivos propios y diferentes del resto, no se está actuando de for- 
ma eficiente. Se están invirtiendo esfuerzos y recursos en conseguir, en ocasiones, objetivos dispares o, aún peor, los mismos objetivos sin que haya flujo de información entre cada subsistema.

- La división entre la formación reglada o inicial y la formación para el empleo, que depende de administraciones diferentes, crea una brecha entre el sistema educativo y el formativo. No hay procesos, herramientas, que guíen a la persona desde la finalización del sistema educativo hasta la entrada al mercado laboral. Su consecuencia más grave, entre otras, son las altas tasas de paro en personas demandantes de su primer empleo.

- Al concebir de forma fragmentada el sistema de formación profesional, los procesos que dan servicio a todo el sistema no están bien desarrollados. Son varias las administraciones que están implicadas en la formación de las personas. Cada una de ellas está ubicada en la base de cada subsistema. En ocasiones, en un mismo subsistema hay varias administraciones con competencias diferentes por territorio. Por tanto, ¿qué ocurre cuando un proceso afecta a varias partes del sistema? Se habla, entre otros temas, de la orientación profesional, la acreditación de competencias profesionales y la prospección de las necesidades formativas de las empresas. La respuesta es que no funcionan o funcionan de forma fragmentada e ineficiente. Cada subsistema puede tener un servicio de orientación profesional, pero si no es transversal, sino hay un procedimiento común, no sirve de nada.

¿En qué se traduce todo lo que se ha expuesto?

- La concepción escolar de todo el sistema de formación profesional da lugar a una elevada burocratización del sistema. La regulación de la formación profesional trata prioritariamente aspectos organizativos o de provisión de la formación, lo que facilita que se releguen a un segundo plano aspectos realmente sustanciales, como los relacionados con la cualificación y el empleo.

- Falta de gobernanza social. La gobernanza social solo se ha restringido al ámbito de la formación profesional para el empleo y solo para tratar tema de los fondos económicos y su gestión.

Esto no ha hecho más que alejar, aún más si cabe, a las empresas del sistema de formación profesional, lo que ha propiciado que se configure como un entorno ajeno al empleo. 


\subsection{La formación profesional dual como parche para integrar el sistema de formación profesional}

En este caldo de cultivo, se publica la ley que regula el contrato de formación y aprendizaje, y de forma tangencial, la formación profesional dual, que viene a asentarse en un sistema de formación profesional que, como ya se ha comentado, adolece per se de problemas estructurales.

La formación profesional dual no es un sistema diferente. Únicamente se trata de una modalidad de oferta distinta dentro de la formación profesional. Su objetivo es que los contenidos de los currículos de los grados medios y superiores no se transmitan exclusivamente de forma teórica, es decir, que se adquieran únicamente en el centro educativo. Entra un nuevo actor en el modelo: la empresa, que se encargará de una forma práctica, a través del trabajo, de enseñarle al aprendiz parte de los contenidos del currículo. Se trata de ir un paso más allá de las meras prácticas en las empresas, en las que el alumno aplica lo que ha aprendido en el centro educativo. En la formación profesional dual se reconoce académicamente lo que la empresa le enseña al aprendiz.

Se han puesto en marcha iniciativas piloto de formación profesional dual, como el proyecto Alianza para la formación profesional dual, en el que Fomento del Trabajo participa. La Fundación Bertelsmann, la CEOE, la Fundación Princesa de Girona y la Cámara de Comercio de España promovieron esta iniciativa para, entre otros objetivos, crear una red estatal de empresas, centros e instituciones comprometidos con el desarrollo de una formación profesional dual de calidad en España. Uno de los primeros servicios que pusieron en marcha los promotores de este proyecto - del cual se forma parte - es la asesoría técnica, cuya función es aclarar dudas que puedan surgir sobre este modelo y apoyar la puesta en marcha de nuevas iniciativas. Así, se empezaron a visitar organizaciones empresariales sectoriales, territoriales, y empresas para poder valorar la situación actual de este modelo en Cataluña y la opinión de empresas y organizaciones al respecto.

Así, se pudo comprobar que la implementación de la formación profesional dual suaviza alguno de los ya mencionados defectos del sistema. Algunos de los logros observados son los siguientes:

- Las empresas que han puesto en marcha una iniciativa de formación profesional dual estrechan vínculos con los aprendices, lo que permite que se transmitan no solo conocimientos profesionales, sino algo mucho más profundo e importante: los valores y la cultura de la empresa. Para las empresas, tener una cantera de profesionales formada por ellos mismos es 
un aspecto valioso. ¿Puede haber algo mejor para la selección de recursos humanos que poder conocer y adecuar al futuro trabajador a su forma de hacer, a su maquinaria, a sus instalaciones, etc.?

+ Las empresas vuelven a su antiguo rol como formadoras - que no debieron abandonar-, lo que facilita, incluso, que puedan llegar a adaptar los currículos a sus necesidades reales.

- Los formadores estrechan vinculos con las empresas. Entre otras formas a través de las cuales puede materializarse esta colaboración, en Cataluña, por ejemplo, el sistema educativo prevé estancias de los formadores en las empresas para que puedan hacer uso de su maquinaria e instalaciones.

- Los participantes están más motivados. Tener la oportunidad de «aprender haciendo» estimula más. Por ejemplo, en los casos en los que no hay plazas de formación profesional dual para todo un grupo de alumnos y los criterios de selección son la nota del alumnado, se propicia que se eleve la media de las calificaciones obtenidas en esa clase.

En realidad, lo que está ocurriendo es que las empresas encuentran en esta modalidad una forma de acercar la formación a sus necesidades reales y, a su vez, con su participación en el sistema de formación profesional lo prestigian.

\section{Viabilidad de la formación profesional dual en España}

No obstante, ¿puede realmente funcionar la formación profesional dual en nuestro país? Se han vertido más ríos de tinta sobre la formación profesional dual que esfuerzos reales en su implementación. Se discute sobre si tiene que ser o no con contrato, qué tipo de contrato tendría que utilizarse, si tiene que estar o no remunerada, cómo tiene que ser la formación de los tutores, si solo es aprendizaje o hay producción, etc. Incluso se invierte mucho tiempo en debates filosóficos sobre lo que puede considerarse formación profesional dual y lo que no. Sin embargo, se está obviando un tema crucial: la cultura que lo fundamenta o lo hace posible. Un marco jurídico que diseñe su aplicación y unos recursos que lo hagan factible son requisitos para que esta formación funcione. Igual de importante es la sensibilización, difusión e información, a fin de tratar de cambiar la concepción que los agentes implicados directa o indirectamente tienen de la formación profesional dual en particular y de la FP en general.

A principios de julio se tuvo la gran oportunidad de ser invitados por el Ministerio Federal de Asuntos Exteriores del Lander de Baden-Württemberg, a través de la Embajada de Alemania en España. Junto con otras personas destacadas 
en el mundo de la formación profesional se pudo conocer in situ la formación profesional dual alemana, en este caso, la de Stuttgart. Durante este encuentro, pudo constatarse lo que se ha afirmado en los párrafos anteriores, además de otros aspectos que se mencionarán a continuación.

Varios estudios señalan la imposibilidad de importar el modelo alemán a España al centrarse en el hecho de que el tejido empresarial es diferente entre estos países, sobre todo en lo que concierne a la medida o al tamaño de las empresas. También intentan fundamentar su hipótesis estructuralmente y, por ello, se basan en la diferencia de instancias y organismos que forman parte del modelo dual tanto en España como en Alemania. Pocos documentos ahondan en la diferencia cultural que hay entre ambos países como una posible causa de la imposibilidad de implantar este modelo.

El modelo alemán funciona al revés del que se está implantando en España. El motor del sistema alemán son las empresas. Las empresas ponen a disposición del sistema plazas de aprendizaje y son los futuros aprendices los que antes de inscribirse en el centro educativo tienen que presentar su currículum en aquellas empresas en las que quieran formarse. También las empresas son las encargadas de elegir a sus futuros aprendices a través de un proceso de selección igual o más estricto que el que se les realiza a otros trabajadores de la empresa. Realmente, es una forma bastante evidente de hacer encajar la oferta y la demanda laboral, que es uno de los problemas presentes en nuestro país y que, de momento, no ha podido solucionarse.

Partiendo de esta premisa, se plantean algunas preguntas: en nuestro país, ¿el sistema educativo forma a los alumnos de enseñanza secundaria en las competencias necesarias para acceder a un proceso de selección de personal con visos de superarlo? ¿Las empresas en España tienen a su alcance los medios necesarios para entrar en el modelo de formación profesional dual? En Alemania hay una mentalidad de sector. Es más relevante la concepción que tienen las empresas sobre el bien común que hacen al mercado laboral y a la sociedad al formar a los aprendices, lo que eleva la profesionalidad del sector, que otros tipos de retornos del sistema.

En Alemania, los centros de formación están comprometidos con el sistema. Tanto los directores de los centros como los formadores se implican al máximo en la formación de los aprendices. Por ejemplo, en la escuela de formación Paul Kerschensteiner pueden observarse fotografías de los aprendices que por allí han pasado y hecho carrera en su profesión, la hostelería, y leerse una pequeña biografía sobre sus logros profesionales. Actualmente, muchos de ellos son cocineros reconocidos internacionalmente. 
Está claro que los recursos económicos que ponen al alcance del sistema son mucho mayores que en España. Los gobiernos, a todos los niveles competenciales, están comprometidos con el sistema. Las empresas donan material y maquinaria a los centros y los aprendices sienten que están en un sistema de prestigio.

Por tanto, la primera y más importante diferencia entre ambos países es un tema de implicación y cultural. En cuanto al modelo, también hay diferencias sustanciales, pero si, primero, no se cree en él de forma general, se considera que será imposible que la formación profesional dual salga adelante en España.

A continuación, se detallarán otros elementos del modelo que se consideran muy interesantes y a los cuales no se les presta demasiada atención porque puede ser que no se conciban como temas sustanciales del modelo.

\subsection{Flexibilidad}

En las dos empresas que se visitaron, los aprendices pasaban por todas las áreas y sus departamentos.

Se visitó un concesionario de coches en el que no importaba que estuvieras estudiando para ser vendedor porque, aun así, pasarías por atención al cliente, venta y mantenimiento, reventa y taller.

También se visitó un hotel de alta categoría, que funcionaba igual. Independientemente de a qué se fuera a dedicar finalmente el aprendiz profesionalmente, tenía que pasar por todas las áreas del hotel, desde la atención al cliente, pasando por el bar, hasta la limpieza de las habitaciones. En cada área lograban fijar aprendizajes distintos. Por ejemplo, en la recepción podían aprender a tratar quejas, a hablar por teléfono, a realizar ventas y a impresionar al cliente. Es una manera de trasladar al aprendiz la cultura de empresa y a enseñarle tareas administrativas relacionadas con ese sector.

\subsection{Sistema de acreditación de competencias profesionales mediante la experiencia laboral o la formación no formal}

Otro tema que han solucionado de forma sencilla es el procedimiento para acreditar las competencias profesionales. Esta es una de las piezas que aún no se consiguen encajar del todo en el sistema de formación profesional español. En España y Cataluña el proceso está muy burocratizado y no es nada ágil. Tanto es así que hablar de un proceso permanente de acreditación de competencias parece una utopía si no se reforma el proceso actual. El Real Decreto 1224/2009, de 17 de julio, de reconocimiento de las competencias profesionales adquiridas por la 
experiencia laboral desarrolla el procedimiento de evaluación y acreditación de las competencias profesionales.

En Alemania, simplemente con un examen - semejante al que pasan los aprendices de formación profesional dual - la persona puede certificar sus competencias profesionales adquiridas por su experiencia laboral. En España, es necesario pasar un proceso que puede durar meses, que se inicia con una fase de asesoramiento y que finaliza con el paso del candidato por una comisión evaluadora, formada por profesores y expertos en la materia que tienen que evaluar, mediante preguntas o pruebas prácticas, el conocimiento de la persona evaluada en las unidades de competencia de las que quiere acreditarse. A esto hay que añadirle el elevado coste del procedimiento, que hasta el momento solo permitía abrir procesos públicos para profesiones en las que era obligatorio presentar una acreditación concreta para poder trabajar. En Cataluña, en los últimos dos años, se ha avanzado en este tema gracias a los acuerdos marco que los agentes sociales han firmado con las administraciones competentes.

\section{Conclusiones}

Se considera que la formación profesional dual tiene aspectos positivos y que es una buena metodología de aprendizaje si se implanta correctamente. Hoy falta mucha información cualitativa y cuantitativa sobre los resultados de las iniciativas finalizadas. Es muy importante que el modelo que se implante sea de calidad para evitar consecuencias negativas sobre el sistema de formación profesional.

Por otra parte, la formación profesional dual no puede ser un parche que suavice los problemas que per se tiene el sistema de formación profesional español. Hay que reformar el sistema, cuyo centro deben ser las empresas y las personas, con la organización y priorización de otros de sus aspectos que no son acciones formales de formación, pero que tienen una importancia capital, como la orientación profesional, la prospección de las necesidades formativas de las empresas y los procesos de acreditación de competencias derivados de la experiencia profesional y la formación no formal. 


\section{Bibliografía}

Comisión Europea (2010): Una estrategia para un crecimiento inteligente, sostenible e integrador, comunicación de la Comisión Europa 2020. Disponible en el siguiente enlace: <eur-lex.europa.eu/LexUriServ/LexUriServ.do?uri=CO M:2010:2020:FIN:ES:PDF>. [Consultado el 24/08/2016].

Convenio marco de colaboración firmado en junio de 2014 por la Generalitat de Cataluña, a través del SOC y el Departamento de Enseñanza, con Fomento del Trabajo Nacional y Fepime-Catalunya para facilitar la acreditación de la experiencia laboral y los aprendizajes no formales de los trabajadores de las empresas asociadas y de sus organizaciones sectoriales y territoriales. Disponible en el siguiente enlace: <www.foment.com/noticias/Paginas/certificacion-acreditacion-competencias-profesionales.asp $x>$.

Convenio entre la Fundación Bertelsmann, la CEOE, la Cámara de España y la Fundación Princesa de Girona con el objetivo de crear una red estatal de instituciones y empresas comprometidas con el desarrollo de la formación profesional dual de calidad en España. Disponible en el siguiente enlace: $<$ www.ceoe.es/es/contenido/actualidad/noticias/nace-la-alianza-para-laformacion-profesional-dual $>$.

Societat d’Estudis Econòmics (2014): El sistema educatiu a Catalunya. Recuperar el prestigi social, Barcelona: Foment del Treball Nacional. 